International Journal of Pure and Applied Mathematics

Volume 113 No. 3 2017, 511-522

ISSN: 1311-8080 (printed version); ISSN: 1314-3395 (on-line version)

url: http://www.ijpam.eu

doi: 10.12732 /jpam.v113i3.13

\title{
SOME PROPERTIES OF $L$-FUZZY PREUNIFORMITIES, $L$-FUZZY NEIGHBORHOOD SYSTEMS AND $L$-FUZZY TOPOLOGIES
}

\author{
Ju-Mok $\mathrm{Oh}^{1}$, A.A.Ramadan ${ }^{2}$, Yong Chan $\mathrm{Kim}^{3} \S$ \\ 1,3 Department of Mathematics \\ Gangneung-Wonju University \\ Gangneung, Gangwondo 25457, KOREA \\ ${ }^{2}$ Mathematics Department \\ Faculty of Science \\ Beni-Suef University \\ Beni-Suef, Egypt
}

\begin{abstract}
This article gives results on $L$-fuzzy preuniformities, $L$-fuzzy neighborhood systems and $L$-fuzzy topologies in complete residuated lattices, and includes some properties. The notion of their continuity property is investigated
\end{abstract}

AMS Subject Classification: 03E72, 06A15, 06F07, 54A05.

Key Words: Complete residuated lattice, $L$-fuzzy neighborhood space, $L$-fuzzy preuniform space, $L$-fuzzy topologies.

\section{Introduction}

Hájek [7] introduced a complete residuated lattice which is an algebraic structure for many valued logic. Bělohlávek [2] investigated information systems and decision rules in complete residuated lattices. Höhle and S.E. Rodabaugh [8] introduced $L$-fuzzy topologies with algebraic structures $L$ (stsc-quantales, MValgebras) as an extension for a completely distributive lattice $L$ or the unit

Received: January 20, 2017

Revised: $\quad$ March 3, 2017

Published:

April 3, 2017
(C) 2017 Academic Publications, Ltd. url: www.acadpubl.eu

${ }^{\S}$ Correspondence author 
interval or the two-point lattice $2=\{0,1\}$. Hutton [9] introduced the notion of fuzzy uniformities in a completely distributive lattice.

Fuzzy uniformities have the following different approaches as follows the entourage approach of Lowen [15,16] , the uniform covering approach of Kotzé [13] and the unification approach of Hutton [9] based on the powersets of the form $\left(L^{X}\right)^{\left(L^{X}\right)}$, the unification approach of Gutiérrez García[6]. Recently, Gutiérrez García introduced $L$-valued Hutton uniformity where a quadruple $(L, \leq, \otimes, *)$ is defined by a $G L$-monoid $(L, *)$ as an extension of a completely distributive lattice $L$. Many researchers studied the different approach as powerset [9] or the uniform covering [13]. Using the Lowen neighborhood system [15], Katsaras [10] proved that every linear fuzzy neighborhood space is uniformizable in the sense of Lowen uniformity. Kim [11] introduced the notion of $L$-fuzzy uniformities as an extension of Lowen in a strictly two-sided, commutative quantale.

This article gives results on $L$-fuzzy preuniformities, stratified $L$-neighborhood systems and $L$-fuzzy topologies in complete residuated lattices, and includes some properties. The notion of their continuity property is investigated

\section{Preliminaries}

Definition 2.1. $[2,4]$ An algebra $(L, \wedge, \vee, \odot, \rightarrow, \perp, \top)$ is called a complete residuated lattice if it satisfies the following conditions:

(C1) $L=(L, \leq, \vee, \wedge, \perp, \top)$ is a complete lattice with the greatest element $\top$ and the least element $\perp$;

(C2) $(L, \odot, \top)$ is a commutative monoid;

(C3) $x \odot y \leq z$ iff $x \leq y \rightarrow z$ for $x, y, z \in L$.

An $L$-subset on a set $X$ is a mapping from $X$ to $L$, and the family of all $L$-subsets on $X$ will be denoted by $L^{X}$; in particular, the $L$-subsets $\top_{X}$ and $\perp_{X}$ defined by $\top_{X}(x)=\top$ and $\perp_{X}(x)=\perp, \quad \forall x \in X$, are respectively the universal upper and lower bound in $L^{X}$. We denote the characteristic function of a subset $\{x\}$ of $X$ by $\top_{x}$. We do not distinguish between an element $\alpha \in L$ and the constant function $\alpha: X \rightarrow L$ such that $\alpha(x)=\alpha$ for all $x \in X$.

All algebraic operations on $L$ can be extended pointwise to the power set $L^{X}$. That is, for all $\lambda, \mu \in L^{X}, \alpha \in L$ and $x \in X$,

(1) $\lambda \leq \mu$ if and only if $\lambda(x) \leq \mu(x)$,

(2) $(\lambda \odot \mu)(x)=\lambda(x) \odot \mu(x)$,

(3) $(\alpha \odot \lambda)(x)=\alpha \odot \lambda(x)$, 
(4) $(\alpha \rightarrow \lambda)(x)=\alpha \rightarrow \lambda(x),(\alpha \odot \lambda)(x)=\alpha \odot \lambda(x)$.

In this paper, we assume that $(L, \vee, \wedge, \odot, \rightarrow, \perp, \top)$ is a complete residuated lattice.

Lemma 2.2. $[2,7,25]$ For each $x, y, z, w, x_{i}, y_{i} \in L$, the following properties hold.

(1) If $y \leq z$, then $x \odot y \leq x \odot z$.

(2) If $y \leq z$, then $x \rightarrow y \leq x \rightarrow z$ and $z \rightarrow x \leq y \rightarrow x$.

(3) $x \rightarrow y=\top$ iff $x \leq y$.

(4) $x \rightarrow \top=\top$ and $\top \rightarrow x=x$.

(5) $x \odot y \leq x \wedge y$.

(6) $x \odot\left(\bigvee_{i \in \Gamma} y_{i}\right)=\bigvee_{i \in \Gamma}\left(x \odot y_{i}\right)$ and $\left(\bigvee_{i \in \Gamma} x_{i}\right) \odot y=\bigvee_{i \in \Gamma}\left(x_{i} \odot y\right)$.

(7) $x \rightarrow\left(\bigwedge_{i \in \Gamma} y_{i}\right)=\bigwedge_{i \in \Gamma}\left(x \rightarrow y_{i}\right)$ and $\left(\bigvee_{i \in \Gamma} x_{i}\right) \rightarrow y=\bigwedge_{i \in \Gamma}\left(x_{i} \rightarrow y\right)$. $\left.y_{i}\right)$.

(8) $\bigvee_{i \in \Gamma} x_{i} \rightarrow \bigvee_{i \in \Gamma} y_{i} \geq \bigwedge_{i \in \Gamma}\left(x_{i} \rightarrow y_{i}\right)$ and $\bigwedge_{i \in \Gamma} x_{i} \rightarrow \bigwedge_{i \in \Gamma} y_{i} \geq \bigwedge_{i \in \Gamma}\left(x_{i} \rightarrow\right.$

(9) $(x \rightarrow y) \odot x \leq y$ and $(x \rightarrow y) \odot(y \rightarrow z) \leq(x \rightarrow z)$.

(10) $x \rightarrow y \leq(y \rightarrow z) \rightarrow(x \rightarrow z)$ and $x \rightarrow y \leq(z \rightarrow x) \rightarrow(z \rightarrow y)$.

(11) $(x \odot y) \rightarrow z=x \rightarrow(y \rightarrow z)=y \rightarrow(x \rightarrow z)$.

(12) $y \rightarrow z \leq x \odot y \rightarrow x \odot z$ and $(x \rightarrow z) \odot(y \rightarrow w) \leq x \odot y \rightarrow z \odot w$.

Lemma 2.3. [3,4] For a given set $X$, define a binary mapping $S: L^{X} \times$ $L^{X} \rightarrow L$ by

$$
S(\lambda, \mu)=\bigwedge_{x \in X}(\lambda(x) \rightarrow \mu(x)) .
$$

Then, for each $\lambda, \mu, \rho, \nu \in L^{X}$, and $\alpha \in L$, the following properties hold.

(1) $S$ is an $L$-partial order on $L^{X}$.

(2) $\lambda \leq \mu$ iff $S(\lambda, \mu) \geq \top$,

(3) If $\lambda \leq \mu$, then $S(\rho, \lambda) \leq S(\rho, \mu)$ and $S(\lambda, \rho) \geq S(\mu, \rho)$,

(4) $S(\lambda, \mu) \odot S(\nu, \rho) \leq S(\lambda \odot \nu, \mu \odot \rho)$,

(5) Let $\phi: X \rightarrow Y$ be an ordinary mapping. Define $\phi^{\rightarrow}: L^{X} \rightarrow L^{Y}$ and $\phi^{\leftarrow}: L^{Y} \rightarrow L^{X}$ by

$$
\begin{aligned}
& \phi^{\rightarrow}(\lambda)(y)=\bigvee_{\phi(x)=y} \lambda(x), \quad \forall \lambda \in L^{X}, y \in Y, \\
& \phi^{\leftarrow}(\mu)(x)=\mu(\phi(x))=\mu \circ \phi(x), \quad \forall \mu \in L^{Y} .
\end{aligned}
$$

Then for $\lambda, \mu \in L^{X}$ and $\rho, \nu \in L^{Y}$,

$$
S(\lambda, \mu) \leq S\left(\phi^{\rightarrow}(\lambda), \phi^{\rightarrow}(\mu)\right)
$$




$$
S(\rho, \nu) \leq S\left(\phi^{\leftarrow}(\rho), \phi^{\leftarrow}(\nu)\right),
$$

and the equalities hold if $\phi$ is bijective.

Definition 2.4. [8] A map $\mathcal{T}: L^{X} \rightarrow L$ is called an $L$-fuzzy topology on $X$ if it satisfies the following conditions.

(O1) $\mathcal{T}\left(\perp_{X}\right)=\mathcal{T}\left(\top_{X}\right)=T$

(O2) $\mathcal{T}(\lambda \odot \mu) \geq \mathcal{T}(\lambda) \odot \mathcal{T}(\mu), \quad \forall \lambda, \mu \in L^{X}$,

(O3) $\mathcal{T}\left(\bigvee_{i} \lambda_{i}\right) \geq \bigwedge_{i} \mathcal{T}\left(\lambda_{i}\right), \quad \forall\left\{\lambda_{i}\right\}_{i \in \Gamma} \subseteq L^{X}$.

An $L$-fuzzy topology is called enriched if

(R) $\mathcal{T}(\alpha \odot \lambda) \geq \mathcal{T}(\lambda)$ for all $\lambda \in L^{X}$ and $\alpha \in L$.

The pair $(X, \mathcal{T})$ is called an $L$-fuzzy topological space.

Let $\left(X, \mathcal{T}_{1}\right)$ and $\left(Y, \mathcal{T}_{2}\right)$ be two $L$-fuzzy topological spaces. A mapping $\phi$ : $X \rightarrow Y$ is said to be fuzzy continuous iff for each $\lambda \in L^{Y}$,

$$
\mathcal{T}_{2}(\lambda) \leq \mathcal{T}_{1}\left(\phi^{\leftarrow}(\lambda)\right) .
$$

Definition 2.5. [8] An $L$-fuzzy neighborhood system on $X$ refers to a collection of mappings $\mathcal{P}=\left\{p_{x}: L^{X} \rightarrow L \mid x \in X\right\}$ such that for any $\lambda, \mu \in L^{X}$ and $x \in X$

(P1) $p_{x}\left(\top_{X}\right)=\top$,

(P2) $p_{x}(\lambda \odot \mu) \geq p_{x}(\lambda) \odot p_{x}(\mu)$

(P3) If $\lambda \leq \mu$, then $p_{x}(\lambda) \leq p_{x}(\mu)$,

(P4) $p_{x}(\lambda) \leq \lambda(x)$ for all $\lambda \in L^{X}$.

An $L$-fuzzy neighborhood system is called stratified if

(R) $p_{x}(\alpha \odot \lambda) \geq \alpha \odot p_{x}(\lambda)$ for all $\lambda \in L^{X}, x \in X$ and $\alpha \in L$.

The pair $(X, \mathcal{P})$ is called an $L$-fuzzy neighborhood space.

Let $(X, \mathcal{P})$ and $(Y, \mathcal{Q})$ be two $L$-fuzzy neighborhood spaces. A mapping $\phi: X \rightarrow Y$ is said to be fuzzy continuous at $x \in X$ iff $q_{\phi(x)}(\lambda) \leq p_{x}\left(\phi^{\leftarrow}(\lambda)\right)$ for each $\lambda \in L^{Y}, \phi$ is continuous if it is fuzzy continuous at every $x \in X$, where $p_{x} \in \mathcal{P}$ and $q_{\phi(x)} \in \mathcal{Q}$.

Theorem 2.6. [12] Let $(X, \mathcal{P})$ be an $L$-fuzzy neighborhood space. Define a map $\mathcal{T}_{\mathcal{P}}: L^{X} \rightarrow L$ by:

$$
\mathcal{T}_{\mathcal{P}}(\lambda)=\bigwedge_{x \in X}\left(\lambda(x) \rightarrow p_{x}(\lambda)\right)=S\left(\lambda, p_{x}(\lambda)\right) .
$$

Then, $\mathcal{T}_{\mathcal{P}}$ is an $L$-fuzzy topology on $X$. If $\mathcal{P}$ is stratified, then $\mathcal{T}_{\mathcal{P}}$ is an enriched $L$-fuzzy topology. 
Theorem 2.7. [12] If a mapping $\phi:(X, \mathcal{P}) \rightarrow(Y, \mathcal{Q})$ is fuzzy continuous, then $\phi:\left(X, \mathcal{T}_{\mathcal{P}}\right) \rightarrow\left(Y, \mathcal{T}_{\mathcal{Q}}\right)$ is fuzzy continuous.

Theorem 2.8. [12] Let $(X, \mathcal{T})$ be an $L$-fuzzy topological space. Define a mapping $\mathcal{P}^{\mathcal{T}}: X \rightarrow L^{L^{X}}$ as follows:

$$
\left(\mathcal{P}^{\mathcal{T}}(x)=p_{x}^{\mathcal{T}}\right)(\lambda)=\bigvee_{\mu \in L^{X}}(\mathcal{T}(\mu) \odot S(\mu, \lambda) \odot \mu(x)) .
$$

Then $\left(X, \mathcal{P}^{\mathcal{T}}\right)$ is a stratified $L$-fuzzy neighborhood space.

Theorem 2.9. [12] Let $\left(X, \mathcal{T}_{X}\right)$ and $\left(Y, \mathcal{T}_{Y}\right)$ be $L$-fuzzy topological spaces. If a mapping $\phi:\left(X, \mathcal{T}_{X}\right) \rightarrow\left(Y, \mathcal{T}_{Y}\right)$ is $L$-fuzzy continuous, then $\phi:\left(X, \mathcal{P}^{\mathcal{T}_{X}}\right) \rightarrow$ $\left(Y, \mathcal{P}^{\mathcal{T}_{Y}}\right)$ is fuzzy continuous.

\section{3. $L$-Fuzzy Preuniformities Induced by $L$-Fuzzy Neighborhood Systems and $L$-Fuzzy Topologies}

Definition 3.1. [6, 8] A map $\mathcal{U}: L^{X \times X} \rightarrow L$ is called an $L$-fuzzy preuniformity on $X$ iff the following conditions hold.

(U1) There exists $u \in L^{X \times X}$ such that $\mathcal{U}(u)=\top$.

(U2) If $v \leq u$, then $\mathcal{U}(v) \leq \mathcal{U}(u)$.

(U3) For every $u, v \in L^{X \times X}, \mathcal{U}(u \odot v) \geq \mathcal{U}(u) \odot \mathcal{U}(v)$.

(U4) $\mathcal{U}(u) \leq S\left(\top_{\triangle}, u\right)=\bigwedge_{x \in X} u(x, x)$, where

$$
\top_{\triangle}(x, y)= \begin{cases}\top, & \text { if } x=y \\ \perp, & \text { if } x \neq y\end{cases}
$$

(U5) $\mathcal{U}(u) \leq \mathcal{U}\left(u^{-1}\right)$, where $u^{-1}(x, y)=u(y, x)$.

An $L$-fuzzy preuniformity $\mathcal{U}$ on $X$ is said to be stratified if

(R) $\mathcal{U}(\alpha \odot u) \geq \alpha \odot \mathcal{U}(u), \quad \forall u \in L^{X \times X}, \alpha \in L$.

The pair $(X, \mathcal{U})$ is called an $L$-fuzzy preuniform space.

Let $(X, \mathcal{U})$ and $(Y, \mathcal{V})$ be $L$-fuzzy preuniform spaces, and $\phi: X \rightarrow Y$ ba a mapping. Then $\phi$ is said to be fuzzy preuniformly continuous if $\mathcal{V}(v) \leq$ $\mathcal{U}\left((\phi \times \phi)^{\leftarrow}(v)\right)$, for every $v \in L^{Y \times Y}$.

Remark 3.2. Let $(X, \mathcal{U})$ be an $L$-fuzzy preuniform space. 
(1) By (U1) and (U2), we have $\mathcal{U}\left(\top_{X \times X}\right)=\top$ because $u \leq \top_{X \times X}$ for all $u \in L^{X \times X}$.

(2) Since $\mathcal{U}(u) \leq \mathcal{U}\left(u^{-1}\right) \leq \mathcal{U}\left(\left(u^{-1}\right)^{-1}\right)=\mathcal{U}(u)$, then $\mathcal{U}(u)=\mathcal{U}\left(u^{-1}\right)$.

Lemma 3.3. Let $\mathcal{U}: L^{X \times X} \rightarrow L$ be a map. The following statement are equivalent

(1) For all $u, v \in L^{X \times X}, S(u, v) \leq \mathcal{U}(u) \rightarrow \mathcal{U}(v)$.

(2) If $u \leq v$, then $\mathcal{U}(u) \leq \mathcal{U}(v)$ and $\mathcal{U}(\alpha \odot u) \geq \alpha \odot \mathcal{U}(u), \forall u \in L^{X \times X}$ and $\alpha \in L$.

(3) If $u \leq v$, then $\mathcal{U}(u) \leq \mathcal{U}(v)$ and $\mathcal{U}(\alpha \rightarrow u) \leq \alpha \rightarrow \mathcal{U}(u), \forall u \in L^{X \times X}$ and $\alpha \in L$.

Proof. $(1) \Rightarrow(2)$. If $u \leq v, \top=S(u, v) \leq \mathcal{U}(u) \rightarrow \mathcal{U}(v)$. Hence, $\mathcal{U}(u) \leq$ $\mathcal{U}(v)$. Put $v=\alpha \odot u$, then $\alpha \leq S(u, \alpha \odot u) \leq \mathcal{U}(u) \rightarrow \mathcal{U}(\alpha \odot u)$. Hence, $\alpha \odot \mathcal{U}(u) \leq \mathcal{U}(\alpha \odot u)$.

$(2) \Rightarrow(3)$. Since $\alpha \odot \mathcal{U}(\alpha \rightarrow u) \leq \mathcal{U}(\alpha \odot(\alpha \rightarrow u)) \leq \mathcal{U}(u)$, by Lemma $(2,2)(9)$.

Hence, $\mathcal{U}(\alpha \rightarrow u) \leq \alpha \rightarrow \mathcal{U}(u)$.

$(3) \Rightarrow(1)$. Since $S(u, v) \odot u \leq v$ if and only if $u \leq S(u, v) \rightarrow v$,

$$
\mathcal{U}(u) \leq \mathcal{U}(S(u, v) \rightarrow v) \leq S(u, v) \rightarrow \mathcal{U}(v)
$$

Hence, $S(u, v) \leq \mathcal{U}(u) \rightarrow \mathcal{U}(v)$.

Definition 3.4. For $\lambda \in L^{X}$, we define $u_{\lambda} \in L^{X \times X}$ associated with $\lambda$ by

$$
u_{\lambda}(x, y)= \begin{cases}\top, & \text { if } x=y \\ \lambda(x) \odot \lambda(y), & \text { if } x \neq y\end{cases}
$$

Theorem 3.5. Let $(X, \mathcal{P})$ be an $L$-fuzzy neighborhood space. Define a $\operatorname{map} \mathcal{U}_{\mathcal{P}}: L^{X \times X} \rightarrow L$ by

$$
\mathcal{U}_{\mathcal{P}}(u)=\bigvee_{\lambda \in L^{X}}\left(\bigvee_{x \in X} p_{x}(\lambda) \odot S\left(u_{\lambda}, u\right)\right), \quad \forall u \in L^{X \times X} .
$$

Then $\left(X, \mathcal{U}_{\mathcal{P}}\right)$ is an $L$-fuzzy preuniform space. If $(X, \mathcal{P})$ is stratified, then $\left(X, \mathcal{U}_{\mathcal{P}}\right)$ is stratified.

Proof. (U1) Since $u_{\top_{X}}=\top_{X \times X}, S\left(u_{\top_{X}}, \top_{X \times X}\right)=\top$, we have

$$
\mathcal{U}_{\mathcal{P}}\left(\top_{X \times X}\right) \geq \bigvee_{x \in X} p_{x}\left(\top_{X}\right) \odot \top=\top \odot \top=\top .
$$


(U2) If $u_{1} \leq u_{2}, u_{1}, u_{2} \in L^{X \times X}$, then for any $\lambda \in L^{X}$, we have by Lemma $2.3(3)$

$$
p_{x}(\lambda) \odot S\left(u_{\lambda}, u_{1}\right) \leq p_{x}(\lambda) \odot S\left(u_{\lambda}, u_{2}\right) .
$$

Taking the supremum we get, $\mathcal{U}_{\mathcal{P}}\left(u_{1}\right) \leq \mathcal{U}_{\mathcal{P}}\left(u_{2}\right)$.

(U3) If $\lambda, \rho \in L^{X}, u, v \in L^{X \times X}$, since $u_{\lambda} \odot u_{\rho}=u_{\lambda \odot \rho}$, we have

$$
\begin{aligned}
& \mathcal{U}_{\mathcal{P}}(u) \odot \mathcal{U}_{\mathcal{P}}(v)=\bigvee_{\lambda \in L^{X}}\left(\bigvee_{x \in X} p_{x}(\lambda) \odot S\left(u_{\lambda}, u\right)\right) \\
& \odot \bigvee_{\rho \in L^{X}}\left(\bigvee_{x \in X} p_{x}(\rho) \odot S\left(u_{\rho}, v\right)\right) \\
& \leq \bigvee_{\lambda, \rho \in L^{X}}\left(\bigvee_{x \in X} p_{x}(\lambda) \odot p_{x}(\rho) \odot S\left(u_{\lambda} \odot u_{\rho}, u \odot v\right)\right)
\end{aligned}
$$

(by Lemma 2.3(4))

$$
\begin{aligned}
& \leq \bigvee_{\lambda, \rho \in L^{X}}\left(\bigvee_{x \in X} p_{x}(\lambda \odot \rho) \odot S\left(u_{\lambda \odot \rho}, u \odot v\right)\right) \\
& \leq \bigvee_{\nu \in L^{X}}\left(\bigvee_{x \in X} p_{x}(\nu) \odot S\left(u_{\nu}, u \odot v\right)\right) \\
& =\mathcal{U}_{\mathcal{P}}(u \odot v) .
\end{aligned}
$$

(U4) If $u \in L^{X \times X}$, then

$$
\begin{aligned}
\mathcal{U}_{\mathcal{P}}(u) & =\bigvee_{\lambda \in L^{X}}\left(\bigvee_{x \in X} p_{x}(\lambda) \odot \bigwedge_{(x, y) \in X \times X}\left(u_{\lambda}(x, y) \rightarrow u(x, y)\right)\right) \\
& \leq \bigvee_{\lambda \in L^{X}}\left(\bigvee_{x \in X} p_{x}(\lambda) \odot \bigwedge_{y \in X}\left(u_{\lambda}(y, y) \rightarrow u(y, y)\right)\right) \\
& \leq \bigvee_{\lambda \in L^{X}}\left(\bigvee_{x \in X} \lambda(x) \odot \bigwedge_{y \in X} u(y, y)\right) \\
& \leq \bigwedge_{y \in X} u(y, y)
\end{aligned}
$$

(U5) Let $u \in L^{X \times X}$, then

$$
\begin{aligned}
\mathcal{U}_{\mathcal{P}}(u) & =\bigvee_{\lambda \in L^{X}}\left(\bigvee_{x \in X} p_{x}(\lambda) \odot \bigwedge_{(x, y) \in X \times X}\left(u_{\lambda}(x, y) \rightarrow u(x, y)\right)\right. \\
& =\bigvee_{\lambda \in L^{X}}\left(\bigvee_{x \in X} p_{x}(\lambda) \odot \bigwedge_{(x, y) \in X \times X}\left(u_{\lambda}^{-1}(y, x) \rightarrow u^{-1}(y, x)\right)\right. \\
& =\mathcal{U}_{\mathcal{P}}\left(u^{-1}\right)
\end{aligned}
$$

Let $(X, \mathcal{P})$ be stratified, $u \in L^{X \times X}, \lambda \in L^{X}$ and $\alpha \in L$, we have

$$
\begin{aligned}
\mathcal{U}_{\mathcal{P}}(\alpha \odot u) & =\bigvee\left(\bigvee_{x \in X}\left(p_{x}(\rho) \odot S\left(u_{\rho}, \alpha \odot u\right)\right)\right) \\
& \geq \bigvee\left(\bigvee_{x \in X}\left(p_{x}(\alpha \odot \lambda) \odot S\left(u_{\alpha \odot \lambda}, \alpha \odot u\right)\right)\right) \\
& \geq \bigvee\left(\alpha \odot \bigvee_{x \in X}\left(p_{x}(\lambda) \odot S\left(\alpha \odot u_{\lambda}, \alpha \odot u\right)\right)\right) \\
& \left.\geq \alpha \odot \bigvee_{x \in X}\left(p_{x}(\lambda) \odot S\left(u_{\lambda}, u\right)\right)\right) \\
& =\alpha \odot \mathcal{U}_{\mathcal{P}}(u) .
\end{aligned}
$$


Hence $\left(X, \mathcal{U}_{\mathcal{P}}\right)$ is stratified.

Theorem 3.6. Let $(X, \mathcal{P})$ and $(Y, \mathcal{Q})$ be $L$-fuzzy neighborhood spaces. Let $\phi:(X, \mathcal{P}) \rightarrow(Y, \mathcal{Q})$ be fuzzy continuous. Then $\phi:\left(X, \mathcal{U}_{\mathcal{P}}\right) \rightarrow\left(Y, \mathcal{V}_{\mathcal{Q}}\right)$ is fuzzy preuniformly continuous.

Proof. Since $(\phi \times \phi)^{\leftarrow}\left(v_{\lambda}\right) \geq v_{\phi^{\leftarrow}(\lambda)}$ and $v(\phi(x), \phi(y))=(\phi \times \phi)^{\leftarrow}(v)(x, y)$, we have,

$$
\begin{aligned}
& \mathcal{V}_{\mathcal{Q}}(v)=\bigvee\left(\bigvee_{y \in Y} q_{y}(\lambda) \odot S\left(v_{\lambda}, v\right)\right) \\
& \leq \bigvee\left(\bigvee_{x \in X} q_{\phi(x)}(\lambda) \odot S\left((\phi \times \phi)^{\leftarrow}\left(v_{\lambda}\right),(\phi \times \phi)^{\leftarrow}(v)\right)\right) \\
& \leq \bigvee\left(\bigvee_{x \in X} p_{x}\left(\phi^{\leftarrow}(\lambda)\right) \odot S\left(v_{\phi^{\leftarrow}(\lambda)},(\phi \times \phi)^{\leftarrow}(v)\right)\right) \\
& \leq \mathcal{U}_{\mathcal{P}}\left((\phi \times \phi)^{\leftarrow}(v)\right) .
\end{aligned}
$$

Theorem 3.7. Let $(X, \mathcal{T})$ be an $L$-fuzzy topological space. Define a map $\mathcal{U}_{\mathcal{T}}: L^{X \times X} \rightarrow L$ by

$$
\mathcal{U}_{\mathcal{T}}(u)=\bigvee_{\lambda \in L^{X}} \bigvee_{x \in X}\left(\lambda(x) \odot \mathcal{T}(\lambda) \odot S\left(u_{\lambda}, u\right)\right) .
$$

Then $(1)\left(X, \mathcal{U}_{\mathcal{T}}\right)$ is an $L$-fuzzy preuniform space,

(2) If $(X, \mathcal{T})$ is enriched, then $\mathcal{U}_{\mathcal{T}}(\alpha \odot u) \geq \mathcal{U}_{\mathcal{T}}(u)$ for all $\alpha \in L$ and $u \in$ $L^{X \times X}$.

Proof. (U1) Since $u_{\top_{X}}=\top_{X \times X}$, we have

$$
\mathcal{U}_{\mathcal{T}}\left(\top_{X \times X}\right) \geq \top_{X}(x) \odot \mathcal{T}\left(\top_{X}\right)=\top .
$$

(U2) If $u_{1} \leq u_{2}, u_{1}, u_{2} \in L^{X \times X}$, then

$$
\begin{aligned}
\mathcal{U}_{\mathcal{T}}\left(u_{1}\right) & =\bigvee_{\lambda \in L^{X}} \bigvee_{x \in X}\left(\lambda(x) \odot \mathcal{T}(\lambda) \odot S\left(u_{\lambda}, u_{1}\right)\right) \\
& \leq \bigvee_{\lambda \in L^{X}} \bigvee_{x \in X}\left(\lambda(x) \odot \mathcal{T}(\lambda) \odot S\left(u_{\lambda}, u_{2}\right)\right)=\mathcal{U}_{\mathcal{T}}\left(u_{2}\right)
\end{aligned}
$$

(U3) Let $\lambda, \rho \in L^{X}$, then $u_{\lambda} \odot u_{\rho}=u_{\lambda \odot \rho}$ and

$$
\begin{aligned}
& \mathcal{U}_{\mathcal{T}}(u) \odot \mathcal{U}_{\mathcal{T}}(w)=\bigvee_{\lambda \in L^{X}} \bigvee_{x \in X}\left(\lambda(x) \odot \mathcal{T}(\lambda) \odot S\left(u_{\lambda}, u\right)\right) \\
& \odot \bigvee_{\lambda \in L^{X}} \bigvee_{x \in X}\left(\rho(x) \odot \mathcal{T}(\rho) \odot S\left(u_{\rho}, w\right)\right) \\
& \leq \bigvee_{\lambda \in L^{X}} \bigvee_{x \in X}\left(\lambda(x) \odot \rho(x) \odot \mathcal{T}(\lambda) \odot \mathcal{T}(\rho) \odot S\left(u_{\lambda} \odot u_{\rho}, u \odot w\right)\right) \\
& \leq \bigvee_{\lambda \in L^{X}} \bigvee_{x \in X}\left((\lambda \odot \rho)(x) \odot \mathcal{T}(\lambda \odot \rho) \odot S\left(u_{\lambda \odot \rho}, u \odot w\right)\right) \\
& \leq \mathcal{U}_{\mathcal{T}}(u \odot w) .
\end{aligned}
$$


(U4)

$$
\begin{aligned}
\mathcal{U}_{\mathcal{T}}(u) & =\bigvee_{\lambda \in L^{X}} \bigvee_{x \in X}\left(\lambda(x) \odot \mathcal{T}(\lambda) \odot S\left(u_{\lambda}, u\right)\right) \\
& =\bigvee_{\lambda \in L^{X}} \bigvee_{x \in X}\left(\lambda(x) \odot \mathcal{T}(\lambda) \odot \bigwedge_{(x, y) \in X \times X}\left(u_{\lambda}(x, y) \rightarrow u(x, y)\right)\right) \\
& \leq \bigvee_{\lambda \in L^{X}} \bigvee_{x \in X}\left(\lambda(x) \odot \mathcal{T}(\lambda) \odot \bigwedge_{y \in X}\left(u_{\lambda}(y, y) \rightarrow u(y, y)\right)\right) \\
& \leq \bigvee_{\lambda \in L^{X}}\left(\mathcal{T}(\lambda) \odot \bigwedge_{y \in X} u(y, y)\right) \\
& =\bigwedge_{y \in X} u(y, y) .
\end{aligned}
$$

(U5) Let $u \in L^{X \times X}$, then

$$
\begin{aligned}
\mathcal{U}_{\mathcal{T}}(u) & =\bigvee_{\lambda \in L^{X}} \bigvee_{x \in X}\left(\lambda(x) \odot \mathcal{T}(\lambda) \odot S\left(u_{\lambda}, u\right)\right) \\
& =\bigvee_{\lambda \in L^{X}} \bigvee_{x \in X}\left(\lambda(x) \odot \mathcal{T}(\lambda) \odot S\left(u_{\lambda}^{-1}, u^{-1}\right)\right)=\mathcal{U}_{\mathcal{T}}\left(u^{-1}\right)
\end{aligned}
$$

Finally, let $u \in L^{X \times X}, \lambda \in L^{X}$ and $\alpha \in L$, we have by Lemma 2.3(4),

$$
\begin{aligned}
\mathcal{U}_{\mathcal{T}}(u) & =\bigvee_{\lambda \in L^{X}} \bigvee_{x \in X}\left(\lambda(x) \odot \mathcal{T}(\lambda) \odot S\left(u_{\lambda}, u\right)\right) \\
& \leq \bigvee_{\lambda \in L^{X}} \bigvee_{x \in X}\left(\lambda(x) \odot \mathcal{T}(\lambda) \odot S\left(\alpha \odot u_{\lambda}, \alpha \odot u\right)\right) \\
& =\bigvee_{\lambda \in L^{X}} \bigvee_{x \in X}\left(\lambda(x) \odot \mathcal{T}(\lambda) \odot S\left(u_{\alpha \odot \lambda}, \alpha \odot u\right)\right) \\
& \leq \bigvee_{\lambda \in L^{X}} \bigvee_{x \in X}\left(\lambda(x) \odot \mathcal{T}(\alpha \odot \lambda) \odot S\left(u_{\alpha \odot \lambda}, \alpha \odot u\right)\right) \\
& \leq \bigvee_{\lambda \in L^{X}} \bigvee_{x \in X}\left(\lambda(x) \odot \mathcal{T}(\mu) \odot S\left(u_{\mu}, \alpha \odot u\right)\right) \\
& =\mathcal{U}_{\mathcal{T}}(\alpha \odot u) .
\end{aligned}
$$

Theorem 3.8. Let $\left(X, \mathcal{T}_{X}\right)$ and $\left(Y, \mathcal{T}_{Y}\right)$ be $L$-fuzzy topological spaces. Let $\phi:\left(X, \mathcal{T}_{X}\right) \rightarrow\left(Y, \mathcal{T}_{Y}\right)$ be surjective fuzzy continuous. Then $\phi:\left(X, \mathcal{U}_{\mathcal{T}_{X}}\right) \rightarrow$ $\left(Y, \mathcal{V}_{\mathcal{T}_{Y}}\right)$ is fuzzy preuniformly continuous.

Proof. Since $(\phi \times \phi)^{\leftarrow}\left(v_{\lambda}\right)\left(x_{1}, x_{2}\right)=v_{\lambda}\left(\phi\left(x_{1}\right), \phi\left(x_{2}\right)\right) \geq v_{\phi^{\leftarrow}(\lambda)}\left(x_{1}, x_{2}\right)$ and $v(\phi(x), \phi(y))=(\phi \times \phi)^{\leftarrow}(v)(x, y)$, we have

$$
\begin{aligned}
& \mathcal{V}_{\mathcal{T}_{Y}}(v)=\bigvee_{\lambda \in L^{Y}} \bigvee_{y \in Y}\left(\lambda(y) \odot \mathcal{T}_{Y}(\lambda) \odot S\left(v_{\lambda}, v\right)\right) \\
& \leq \bigvee_{\lambda \in L^{Y}} \bigvee_{y \in Y}\left(\lambda(y) \odot \mathcal{T}_{Y}(\lambda) \odot S\left((\phi \times \phi)^{\leftarrow}\left(v_{\lambda}\right),(\phi \times \phi)^{\leftarrow}(v)\right)\right) \\
& \leq \bigvee_{\lambda \in L^{Y}} \bigvee_{x \in X}\left(\lambda(\phi(x)) \odot \mathcal{T}_{X}\left(\phi^{\leftarrow}(\lambda)\right) \odot S\left(v_{\phi^{\leftarrow}(\lambda)},(\phi \times \phi)^{\leftarrow}(v)\right)\right) \\
& \leq \mathcal{U}_{\mathcal{T}_{X}}\left((\phi \times \phi)^{\leftarrow}(v)\right) .
\end{aligned}
$$


Example 3.9. Let $(L=[0,1], \odot, \rightarrow)$ be a complete residuated lattice defined by

$$
x \odot y=(x+y-1) \vee 0, \quad x \rightarrow y=(1-x+y) \wedge 1 .
$$

Let $X=\{x, y, z\}$ be a set and $\rho \in L^{X}$ with $\rho(x)=0.6, \rho(y)=0.6, \rho(z)=0.5$. Define $\mathcal{T}: L^{X} \rightarrow L$ as follows

$$
\mathcal{T}(\lambda)= \begin{cases}1, & \text { if } \lambda \in\left\{1_{X}, 0_{X}\right\} \\ 0.6, & \text { if } \lambda=\rho \\ 0.3, & \text { if } \lambda=\rho \odot \rho \\ 0, & \text { otherwise }\end{cases}
$$

We obtain $u_{\rho \otimes \rho}=1_{\triangle}$ and

$$
u_{\rho}=\left(\begin{array}{ccc}
1 & 0.2 & 0.1 \\
0.2 & 1 & 0.1 \\
0.1 & 0.1 & 1
\end{array}\right)
$$

By Theorem 3.7, we can construct $\mathcal{U}_{\mathcal{T}}: L^{X \times X} \rightarrow L$ as follows

$$
\mathcal{U}_{\mathcal{T}}(u)=\left(\bigwedge_{(x, y) \in X \times X} u(x, y)\right) \vee\left(0.6 \odot S\left(u_{\rho}, u\right)\right) \vee\left(0.3 \odot \bigwedge_{x \in X} u(x, x)\right) .
$$

By Theorem 2.8, we can obtain $\mathcal{P}^{\mathcal{T}}: X \rightarrow L^{L^{X}}$ as follows

$$
\begin{aligned}
& p_{x}^{\mathcal{T}}(\lambda)=\left(\bigwedge_{x \in X} \lambda(x)\right) \vee(0.6 \odot S(\rho, \lambda) \odot 0.6), \\
& p_{y}^{\mathcal{T}}(\lambda)=\left(\bigwedge_{x \in X} \lambda(x)\right) \vee(0.6 \odot S(\rho, \lambda) \odot 0.6), \\
& p_{z}^{\mathcal{T}}(\lambda)=\left(\bigwedge_{x \in X} \lambda(x)\right) \vee(0.6 \odot S(\rho, \lambda) \odot 0.5) .
\end{aligned}
$$

By Theorem 3.5, we can construct $\mathcal{U}_{\mathcal{P}_{\mathcal{T}}}: L^{X \times X} \rightarrow L$ as follows

$$
\mathcal{U}_{\mathcal{P}_{\mathcal{T}}}(u)=\bigvee_{\lambda \in L^{X}}\left(\left(\bigwedge_{x \in X} \lambda(x) \vee(0.2 \odot S(\rho, \lambda))\right) \odot S\left(u_{\lambda}, u\right)\right)
$$




\section{Acknowledgments}

This work was supported by Research Fund of Gangneung-Wonju National University in 2016.

\section{References}

[1] R. Badard, A.A. Ramadan, A.S. Mashhour, Smooth preuniform and proximity spaces, Fuzzy Sets and Systems, 59 (1993), 95-107.

[2] R. Bělohlávek, Fuzzy Relational Systems, Kluwer Academic Publishers, New York (2002), doi: 10.1007/978-1-4615-0633-1.

[3] Fang Jinming, I-fuzzy Alexandrov topologies and specialization orders, Fuzzy Sets and Systems, 158 (2007), 2359-2374.

[4] J. Fang, The relationship between L-ordered convergence structures and strong Ltopologies, Fuzzy Sets and Systems, 161 (2010), 2923-2944.

[5] J.Gutiérrez García, I. Mardones Peerez, M.H. Burton, The relationship between various filter notions on a GL-monoid, J. Math. Anal. Appl., 230 (1999), 291- 302.

[6] J.Gutiérrez García, M. A. de Prade Vicente, A.P. Šostak, A unified approach to the concept of fuzzy Luniform spaces, Chapter 3, 81-114 in [23].

[7] P. Hájek, Metamathematices of Fuzzy Logic, Kluwer Academic Publishers, Dordrecht, 1998, doi: 10.1007/978-94-011-5300-3.

[8] U. Höhle, S.E. Rodabaugh, Mathematics of Fuzzy Sets: Logic, Topology, and Measure Theory, The Handbooks of Fuzzy Sets Series 3, Kluwer Academic Publishers, Boston (1999), doi: 10.1007/978-1-4615-5079-2.

[9] B. Hutton, Uniformities in fuzzy topological spaces, J. Math. Anal. Appl., 58 (1977), 74-79, doi: 10.1016/0022-247x(77)90192-5.

[10] A.K. Katsaras, On fuzzy uniform spaces, J. Math. Anal. Appl., 101 (1984), 97-113, doi: 10.1016/0022-247x(84)90060-x.

[11] Y.C. Kim, A.A. Ramadan, M. A. Usama, L-fuzzy Uniform Spaces, The Journal of Fuzzy Mathematics, 14 (2006), 821-850.

[12] J.M. Ko, J.M. Oh, Relationships between $L$-neighborhood systems and $L$-fuzzy topologies, Int.J. Pure and Applied Math., 112(3), (2017) 631-643, doi: 10.12732/ijpam.v112i3.14.

[13] W. Kotzé, Uniform spaces, Chapter 8, 553-580 in [8].

[14] T. Kubiak, On fuzzy topologies, Ph.D. Thesis, Adam Mickiewicz Uniformity, Poznan, Poland, (1985)

[15] R. Lowen, Fuzzy uniform spaces, J. Math. Anal. Appl., 82 (1981), 370-385.

[16] R. Lowen, Fuzzy neighborhood spaces, Fuzzy Sets and Systems, 7 (1982), 165-189.

[17] H. Lai, D. Zhang, Fuzzy preorder and fuzzy topology, Fuzzy Sets and Systems, 157 (2006), 1865-1885. 
[18] A.A. Ramadan,Y.C. Kim, M.K. El-Gayyar, On fuzzy uniform spaces, The Journal of Fuzzy Mathematics, 11 (2003), 279-299.

[19] A.A. Ramadan, E.H.Elkordy, Yong Chan Kim, Relationships between $L$-fuzzy quasiuniform stuctures and L-fuzzy topologies, Journal of Intelligent and Fuzzy Systems, 28 (2015), 2319-2327.

[20] A.A. Ramadan, On $L$-fuzzy interior operators and $L$-fuzzy quasi-uniform spaces, Journal of Intelligent and Fuzzy Systems, in press.

[21] S.E. Rodabaugh, A theory of fuzzy uniformities with applications to the fuzzy real lines, J. Math. Anal. Appl., 129 (1988), 37-70.

[22] S.E. Rodabaugh, E.P. Klement, Topological and Algebraic Structures In Fuzzy Sets, The Handbook of Recent Developments in the Mathematics of Fuzzy Sets, Kluwer Academic Publishers, Boston, Dordrecht, London, (2003)

[23] A.P. Šostak, On a fuzzy topological structure, Suppl., Rend Circ. Matem. Palermo, Ser. II [11] (1985), 125- 186.

[24] A.P. Šostak, Towards the theory of M-approximate systems, Fuzzy Sets and Systems, 161 (2010), 2440- 2461.

[25] E. Turunen, Mathematics Behind Fuzzy Logic, A Springer-Verlag Co., Heidelberg (1999). 